\title{
10\% Loss of Incident Power through Solar Reactor Window: Myth or Good Rule of Thumb?
}

\author{
V. Pozzobon \\ Université de Toulouse, France \\ e-mail: victor.pozzobon@mines-albi.fr
}

\begin{abstract}
It is known that a solar beam crossing a window losses $10 \%$ of its incident power. Yet, this affirmation is not supported by many published scientific evidences. In this work, a heat flux mapping method was used to determine the heat flux distributions at the focal spot of a solar concentrating device without and with a window on the incident beams' trajectory. The presence of a window on the beams' trajectory induces a $12 \%$ loss of the total power and a $11 \%$ decrease of the peak heat flux density.
\end{abstract}

\section{INTRODUCTION}

Concentrated solar power can be used to supply heat at high temperature. It features several advantages compared to conventional fossil fuel burning methods. Among them is the fact that concentrated solar power supplies clean heat, i.e., without combustion fumes. Indeed, those fumes may alter or even destroy the heated material, e.g. decomposition of limestone [1]. In addition, whenever the reacting atmosphere needs to be controlled, it is common to add a windowed aperture to the reactor design [2-4]. This window ensures the airtightness of the reactor while allowing the solar heat flux to enter it.

Nevertheless, adding a window comes with one main drawback: it lowers the amount of energy entering the reactor. Indeed, the incident flux crosses the window and therefore loses part of its power because of in medium absorption and dioptres reflections. It is, most of the time, quoted as common knowledge in the field of solar reactor design that crossing a window induces a $10 \%$ loss of the incident power. Yet, only one research paper was found to support this claim, in the very particular case of a dome [5], and not of a flat window. Furthermore, this claim is not complete for it only regards the total power: it does not precise whether or not the heat flux distribution is modified.

In order to assess for the validity of this claim, the heat flux distributions at the focal spot of a solar concentrating system were mapped with and without a quartz window on the beams' trajectory. Then, the total incident power and the shape of the heat flux distribution were compared.

\section{MATERIALS AND METHODS}

Figure 1 shows a schematic of the solar concentrating device, i.e. an artificial sun, and the heat flux measurement material. To map the incident heat flux, a screen is set in front of the artificial sun at the focal spot. Thus, the beams coming out of it are intercepted by the screen. As beams' energy is absorbed by the screen, its temperature rises. The temperature variations are recorded by IR camera. Then by inverse methods using the temperature elevation is used to compute the incident heat flux distribution over the screen. A 2D model is used to link temperature $(T)$ rise with incident heat flux $(\Phi)$. It accounts for the contribution of the incident radiative heat flux as well as convective and radiative heat losses:

$$
\begin{gathered}
\rho c_{p} e \frac{\partial T}{\partial t}=\lambda e \Delta T+\alpha \Phi \\
-2 h\left(T-T_{\text {sur }}\right)-2 \in \sigma\left(T^{4}-T_{\text {sur }}^{4}\right),
\end{gathered}
$$

where $\rho, c_{p}, \lambda, \alpha, \varepsilon, e$ screen density, heat capacity, conductivity, absorptivity, emissivity and thickness respectively, $T_{\text {sur }}$ and $\mathrm{h}$, surrounding temperature and convective heat flux coefficient.

The model is solved for each pixel of the recorded images using ordinary least square method. Once completed, this procedure yields a map of the incident heat flux. The solar concentrating system and the heat flux mapping method used in this work have been extensively described in [6].

A $3 \mathrm{~mm}$ thick flat quartz sheet was used to simulate a reactor window. The window was set $5 \mathrm{~cm}$ above the screen, parallel to it and perpendicular to the system revolution axis. The repeatability of the measured heat flux distributions was assessed by repeating the mea- 


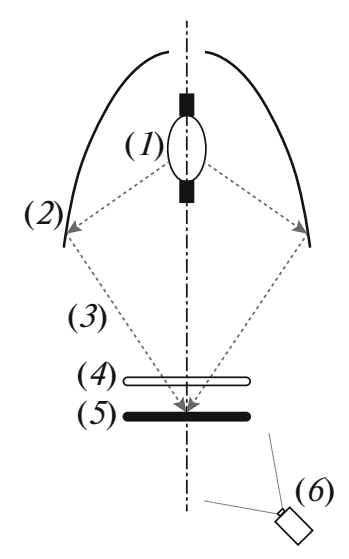

Fig. 1. Experimental apparatus schematics [6]. (1) $4 \mathrm{~kW}$ xenon arc lamp, (2) elliptical mirror, (3) a ray, (4) removable quartz window, (5) screen, (6) camera.

surement twice in both configurations. The repeatability is very good. The absorption of the window was computed with respect to the lamp radiation spec- trum. The quartz window was found to absorb $7.5 \%$ of the incident power.

\section{RESULTS}

Figure 2 reports the determined heat flux distributions at the focal spot without and with the additional quartz window. The spatial distributions are similar; furthermore they both exhibit revolution symmetry. Figure 3 reports cut views of the heat flux distribution along $\mathrm{x}$ and $\mathrm{y}$ axes at the focal spot. In both configurations, the distributions along the two axes are very close. They exhibit a Gaussian shape, which is congruent with literature [7-10].

The peak heat flux was measured to be $1201 \mathrm{~kW} / \mathrm{m}^{2}$ without the window and $1072 \mathrm{~kW} / \mathrm{m}^{2}$ with the window. The window therefore induces an $11 \%$ decrease of the peak heat flux with respect to the unshaded configuration. Furthermore, adding a window leads the total incident power to decrease from $936 \mathrm{~W}$, without the quartz sheet, to $820 \mathrm{~W}$. It represents a $12 \%$ loss of the incident power. Owing regards to the window
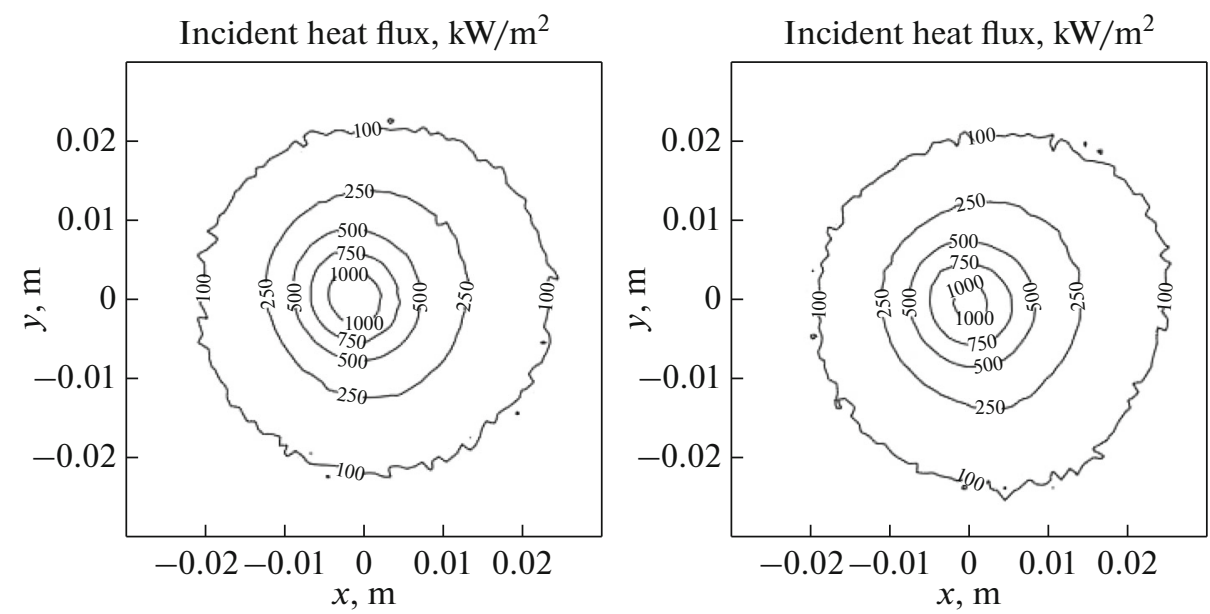

Fig. 2. Heat flux map at the focal spot without additional window (on the left) and with additional window (on the right).
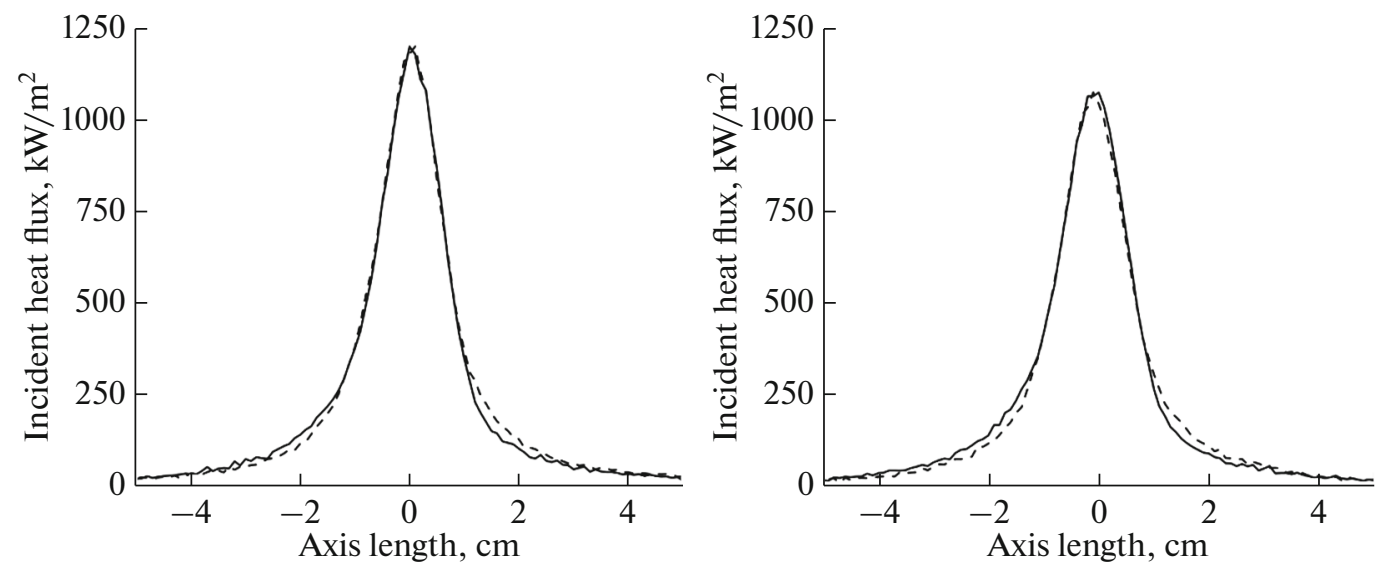

Fig. 3. Heat flux distributions at the focal spot without additional window (on the left) and with additional window (on the right). Continuous line: along $x$ axis, dashed line: along $y$ axis. 


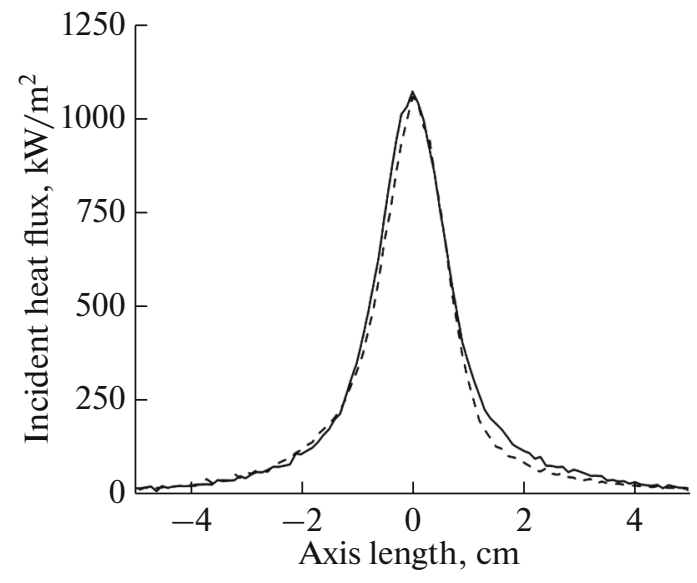

Fig. 4. Heat flux distributions at the focal spot. Continuous line: with additional window, dashed line: without additional window multiplied by 0.88 .

absorption, it can be concluded that reflections induce a $4.5 \%$ loss of the incident power.

In order to assess for the similarity in the shape of the two heat flux distributions, the heat flux distribution along $x$ axis without the windows was multiplied by 0.88 and compared to the one measured along $x$ axis with the window. Figure 4 shows both experimentally observed and computed heat flux distributions. The two distributions are very close. It can therefore be concluded that crossing a window does not modify the shape of the heat flux distribution.

\section{CONCLUSIONS}

This brief note presents heat flux mappings, without and with an intermediate window, at the focal spot of a solar concentrating system. From the experimental measurements, it can be concluded that crossing a window induces a $12 \%$ loss of the incident power and a $11 \%$ reduction of the peak heat flux density. Furthermore, the heat flux distribution shape after crossing the window is almost identical to the one before. The two heat flux distribution shape can be linked by a simple multiplication by a 0.88 factor. As a conclusion, it can be stated that: as a good rule of thumb, one can consider that crossing a window reduces by $12 \%$ the total power and the peak density while conserving the shape of the heat flux distribution.

\section{REFERENCES}

1. Imhof, A., Renewable Energy, 1997, vol. 10, nos. 2-3, pp. 239-246.

2. Z'Graggen, A. and Steinfeld, A., Int. J. Hydrogen Energy, 2008, vol. 33, pp. 5484-5492.

3. Salikhov, T.P., Kan, V.V., and Kan, S.N., Appl. Sol. Energy, 2007, vol. 43, no. 1, pp 54-55.

4. Faiziev, Sh. A., Paizullakhanov, M. S., and Nurmatov, Sh.R., Features of zirconium carbonitride synthesis in a large solar furnace, Appl. Sol. Energy, 2010, vol. 46 , no. 1 , pp. 53-55.

5. Guesdon, C., Alxneit, I., Tschudi, H.R., et al., Sol. Energy, 2006, vol. 80, pp. 1344-1348.

6. Pozzobon, V. and Salvador, S., Sol. Energy, 2015, vol. 117, pp. 29-35.

7. Sarwar, J., Georgakis, G., LaChance, R., and Ozalp, N., Sol. Energy, 2014, vol. 100, pp. 179-194.

8. Petrasch, J., Coray, P., Meier, A., et al., J. Sol. Energy Eng. - Trans. ASME, 2007, vol. 129, pp. 405-411.

9. Llorente, J., Ballestrin, J., and Vazquez, A.J., Sol. Energy, 2011, vol. 85, pp. 1000-1006.

10. Abdurakhmanov, A.A., Klychev, Sh.I., Nurmatov, Sh.R., Faiziev, Sh.A., and Paizullakhanov, M.S., Appl. Sol. Energy, 2009, vol. 45, no. 4, pp. 279-280. 